\title{
The bootstrap for impact factors and the gluon wave function
}

\author{
Mikhail Braun ${ }^{a}$, and Gian Paolo Vacca ${ }^{b}$ \\ a) Department of high-energy physics, University of S. Petersburg \\ ${ }^{b)}$ II. Institute für Theoretische Physik, Universität Hamburg.
}

December 25, 2017

\begin{abstract}
Using the results recently obtained for the non-forward quark and gluon impact factors, it is shown that their form in the gluon colour channel is consistent with the "third bootstrap condition", namely, that they should be proportional to the gluon wave function. The gluon wave function found from this assumption is used to write the full bootstrap condition for the gluonic potential in the next-to-leading order.
\end{abstract}

\section{Introduction.}

Recently the 2nd order corrections were calculated for the gluon and quark non-forward impact factors $[1,2]$. The authors checked the so-called "second bootstrap condition" for them and found that it was satisfied. There is however still another ("third") bootstrap condition for the impact factors discussed in [3]. It is not operative for the elastic particle-particle (PP) amplitudes but becomes essential if one considers particle-reggeon (PR) amplitudes. Such amplitudes naturally appear if one considers inelastic amplitudes with production of one or several gluons. Fulfillment of the third bootstrap condition seems necessary to satisfy unitarity for inelastic amplitudes [4]

As discussed in [3], the third bootstrap condition is just a requirement that in the gluonic channel the impact factor for ANY particle, considered as a function of reggeonic momenta, coincide with the gluon wave function up to a factor which may depend on the transferred momentum. In this note we check this requirement for the impact factors calculated in $[1,2]$.

From the start it is clear that since the gluon wave function is unknown in the next-to-leading order (NLO), checking of the third bootstrap condition strictly speaking reduces to verifying that both the gluon and quark impact factors have the same dependence on the reggeonic momenta. Our result is that this prediction of the "third bootstrap" is true: the dependence on the reggeonic momenta is indeed identical for the gluon and quark impact factors. Assuming that the "third bootstrap" condition is valid, we are able to find the 2nd order corrections to the gluon wave function and consequently write the main bootstrap condition in the 2 nd order. This is a stringent test for the 2 nd order potential, still unknown at present.

In [5] an ansatz was proposed for the gluon trajectory, wave function and interaction potential to introduce the running coupling to all orders of the fixed one. It solves the main bootstrap condition (the "first" one) identically. It was found in [3] that a part of the NLO interaction in the gluonic channel which comes from an intermediate $q \bar{q}$ pair found from this ansatz coincides with the one found by direct calculation in [6]. So the ansatz works for the part due to an intermediate $q \bar{q}$ pair. However our results show that, if the third bootstrap is fulfilled, the ansatz is not valid for the part of the gluon wave function generated by gluonic intermediate states, although it correctly reproduces the running of the coupling.

\section{The third bootstrap condition}

Consider the non-forward elastic PP amplitude generated by the exchange of two reggeized gluons in the gluon colour channel. Its absorptive part as a function of the angular momentum $j$ and in the 
leading and next-to-leading orders can be written as

$$
\mathcal{A}_{j}=\left\langle\Phi_{p}|G(E)| \Phi_{t}\right\rangle .
$$

Here the functions $\Phi_{p, t}\left(q, q_{1}\right)$ are the impact factors which represent the coupling of the external particles ( $t$ from the target and $p$ from the projectile) to the two exchanged reggeized gluons with momenta $q_{1}$ and $q_{2}=q-q_{1}$, where $q$ is an overall momentum transfer. The 2-gluon Green function $G(E)=(H-E)^{-1}$ with $H$ the Hamiltonian and $E=1-j$, can be represented via the orthonormalized solutions of the homogeneous Schrödinger equation

$$
H \Psi_{n}=E_{n} \Psi_{n} .
$$

As a result, the absorptive part $\mathcal{A}_{j}$ can be written as

$$
\mathcal{A}_{j}=\sum_{n} \frac{\left\langle\Phi_{p} \mid \Psi_{n}\right\rangle\left\langle\Psi_{n} \mid \Phi_{t}\right\rangle}{E_{n}-E} .
$$

We shall be also interested in the PR amplitudes, defined as

$$
\Psi(E)=G(E)|\Phi\rangle,
$$

where $\Phi$ is the particle impact factor. Amplitude $\Psi$ satisfies an inhomogeneous Schroedinger equation

$$
(H-E) \Psi(E)=\Phi .
$$

In terms of the eigenfunctions $\Psi_{n}$ one has

$$
\Psi(E)=\sum_{n} \frac{\Psi_{n}\left\langle\Psi_{n} \mid \Phi\right\rangle}{E_{n}-E} .
$$

The bootstrap conditions come from the requirement that in the gluon colour channel amplitudes generated from their absorptive parts have the form corresponding to the exchange of a single reggeized gluon. From this it follows that the spectrum of $H$ in this colour channel should contain an eigenvalue $E_{0}=-\omega(q)$ here $\omega(q)$ is the gluon Regge trajectory. The corresponding eigenfunction $\Psi_{0}$ is the gluon wave function:

$$
(H+\omega(q)) \Psi_{0}=0 .
$$

Eq. (7) is the first bootstrap condition. The second bootstrap condition, introduced in [7], requires that the residue of the PP amplitude at the gluon Regge pole correspond to its value obtained from (1). In fact this is a condition which constrains the form of the PPR vertex.

There is finally a third requirement. Namely, the amplitude generated from its absorptive part should not contain admixture of other eigenstates of the Hamiltonian different from the gluon. This requires that, as a function of reggeized gluons momenta, the impact factor coincides with the gluonic wave function $\Psi_{0}$ up to a coefficient which may depend on the momentum transfer $q$ :

$$
\Phi\left(q, q_{1}\right)=c(q) \Psi_{0}\left(q, q_{1}\right) .
$$

This is the third bootstrap condition which we are going to discuss in this note.

As pointed in [3], this third bootstrap condition is automatically satisfied in the NLO for the physical amplitude (1). Indeed, all bootstrap conditions are satisfied in the leading order (LO). As a result, the ratio

$$
\left\langle\Psi_{n} \mid \Phi\right\rangle /\left\langle\Psi_{0} \mid \Phi\right\rangle
$$

for states $n \neq 0$ different from the one-gluon starts from the order $\alpha_{s}$. From (3) we see that the relative contribution of states different from the gluon involves a product of two such ratios for the projetile and target and is therefore a correction of order $\alpha_{s}^{2}$, beyond the NLO approximation.

However this is not true for the (unphysical) PR amplitudes, which involve terms proportional to $\left\langle\Psi_{n} \mid \Phi\right\rangle$, of the relative order $\alpha_{s}$. If (8) is not fulfilled in the NLO, then in the NLO PR amplitudes will inevitably contain contributions from states different from the one gluon state. This does not spoil the unitarity for elastic amplitudes, but may invalidate the unitarity for inelastic amplitudes, with production of additional gluons, as discussed in [4]. 
The third bootstrap condition (8) implies that for any two physical particles the ratio of impact factors is independent of the reggeized gluon momenta and so is a function of only momentum transfer $q$

$$
\frac{\Phi_{1}\left(q, q_{1}\right)}{\Phi_{2}\left(q, q_{1}\right)}=F(q)
$$

This is a general statement, which does not depend on a particular form of the gluon wave function.

A more particular form of this condition can be written once the gluonic wave function is known. In [5] an ansatz for it was proposed (in the metric $\int d^{2} q_{1}^{\prime}$ and for an Hermithean Hamiltonian)

$$
\Psi_{0}\left(q, q_{1}\right)=1 / \sqrt{\eta\left(q_{1}\right) \eta\left(q_{2}\right)}, \quad q_{1}+q_{2}=q,
$$

where $\eta(q)$ is a function which can be determined from the gluon trajectory $\omega(q)$ by the equation

$$
\omega(q)=-\eta(q)\left\langle\Psi_{0} \mid \Psi_{0}\right\rangle .
$$

(In fact the gluonic potential in the gluon colour channel is also determined by $\eta$ to satisfy the first bootstrap condition (7), but this is irrelevant here). In the LO

$$
\eta^{(0)}(q)=q^{2} / a, \quad a=\frac{g^{2} N}{2(2 \pi)^{D-1}} .
$$

In the NLO function $\eta(q)$ was calculated in [3]. Later we shall return to explicit form. One can try to verify if (8) is true or not, with the gluonic wave function given by (10),

Presenting the impact factor as

$$
\Phi\left(q, q_{1}\right)=\Phi^{(0)}\left(q, q_{1}\right)\left(1+\Delta\left(q, q_{1}\right)\right),
$$

where term $\Delta$, of the order $\alpha_{s}$, is the NLO correction one gets from (9) a requirement that the difference of $\Delta$ 's should be independent of $q_{1}$ for any two particles:

$$
\Delta_{1}\left(q, q_{1}\right)-\Delta_{2}\left(q, q_{1}\right)=F_{1}(q) .
$$

If we use the specific form (10) for the gluon wave function and present

$$
\eta(q)=\eta^{(0)}(q)(1+\xi(q)),
$$

where again $\xi$ gives the NLO correction, then according to (8) the dependence of $\Delta\left(q, q_{1}\right)$ on $q_{1}$ has to be completely determined by that of $\eta(q)$

$$
\Delta\left(q, q_{1}\right)=-(1 / 2)\left(\xi\left(q_{1}\right)+\xi\left(q_{2}\right)\right)+\zeta(q), \quad q_{1}+q_{2}=q,
$$

where $\zeta$ coming from $c$ in (9) is an unknown function of $q$.

Note that in Eqs. (14) and (16) it is assumed that the impact factor is different from zero in the LO. In fact in the NLO appear helicity changing terms, absent in the LO. For such terms the third bootstrap condition reduces to the requirement that they should be proportional to the LO gluon function, that is, independent of the gluonic momenta.

It is these requirements that we are going to check for the impact factors calculated in $[1,2]$. We restrict ourselves to a simpler case of massless quarks.

\section{The gluon impact factor in the gluon channel}

In this and following sections we reproduce the results of $[1,2]$ for the NLO impact factors in the massless quark case, projected onto the gluon colour channel in slightly different notation adapted to our purpose. The gluon NLO impact factor $\Phi_{g}^{(1)}$ consists of three terms

$$
\Phi_{g}^{(1)}=\Phi_{g 1}^{(1)}+\Phi_{g 2}^{(1)}+\Phi_{g 3}^{(1)}
$$

corresponding to the one-gluon, $q \bar{q}$ and two-gluon intermediate states respectively. Their explicit form is given by the following expressions. The part from the one-gluon state is

$$
\Phi_{g 1}^{(1)}\left(q, q_{1}\right)=\beta \Phi_{g}^{(0)} N\left[q_{1}^{2 \epsilon} \ln \left(\frac{s_{0}}{q_{1}^{2}}\right)+q_{2}^{2 \epsilon} \ln \left(\frac{s_{0}}{q_{2}^{2}}\right)+\left(\frac{2}{\epsilon}-\frac{(11+9 \epsilon)}{2(1+2 \epsilon)(3+2 \epsilon)}\right.\right.
$$




$$
\begin{gathered}
\left.\left.+\frac{n_{f}}{N} \frac{(1+\epsilon)(2+\epsilon)-1}{(1+\epsilon)(1+2 \epsilon)(3+2 \epsilon)}+\psi(1)+\psi(1-\epsilon)-2 \psi(1+\epsilon)\right)\left(q_{1}^{2 \epsilon}+q_{2}^{2 \epsilon}\right)\right] \\
+\beta N\left(\chi\left(q_{1}\right)+\chi\left(q_{2}\right)\right) \frac{2 \epsilon}{(1+\epsilon)(1+2 \epsilon)(3+2 \epsilon)}\left(1+\epsilon-\frac{n_{f}}{N}\right) .
\end{gathered}
$$

Here $\Phi_{g}^{(0)}$ is the impact factor in the LO

$$
\Phi_{g}^{(0)}=i g^{2}\left(e^{\prime} e\right)_{\perp} \frac{\sqrt{N}}{2} T^{a},
$$

with $e$ and $e^{\prime}$ the gluon polarization vectors, $T$ its colour vector and $N$ and $n_{f}$ the numbers of colours and flavours. Function $\chi$ is defined as

$$
\chi\left(q_{1}\right)=g^{2} i \frac{\sqrt{N}}{2} T^{a} q_{1}^{2 \epsilon}\left(e^{\prime} q_{1}\right)\left(e q_{1}\right) / q_{1}^{2}
$$

and the coefficient is

$$
\beta=\frac{g^{2}}{(4 \pi)^{2+\epsilon}} \frac{\Gamma(-\epsilon) \Gamma^{2}(1+\epsilon)}{\Gamma(1+2 \epsilon)},
$$

where $D=4+2 \epsilon$ is the dimension used to calculate the 4-dimensional divergent integrals. The part coming from the $q \bar{q}$ state is

$$
\begin{gathered}
\Phi_{g 2}^{(1)}\left(q, q_{1}\right)=-\beta \Phi_{g}^{(0)} n_{f} \frac{2(1+\epsilon)^{2}+\epsilon}{(1+\epsilon)(1+2 \epsilon)(3+2 \epsilon)}\left(q_{1}^{2 \epsilon}+q_{2}^{2 \epsilon}-q^{2 \epsilon}\right) \\
+\beta n_{f} \frac{2 \epsilon}{(1+\epsilon)(1+2 \epsilon)(3+2 \epsilon)}\left(\chi\left(q_{1}\right)+\chi\left(q_{2}\right)-\chi(q)\right) .
\end{gathered}
$$

Finally the two-gluon intermediate state generates a contribution

$$
\begin{gathered}
\Phi_{g 3}^{(1)}\left(q, q_{1}\right)=-\beta N \Phi_{g}^{(0)}\left[q_{1}^{2 \epsilon} \ln \left(\frac{s_{0}}{q_{1}^{2}}\right)+q_{2}^{2 \epsilon} \ln \left(\frac{s_{0}}{q_{2}^{2}}\right)-q^{2 \epsilon} \ln \left(\frac{s_{0}}{q^{2}}\right)+\right. \\
\left(\frac{3}{2 \epsilon}-\frac{(11+8 \epsilon)}{(1+2 \epsilon)(3+2 \epsilon)}-\psi(1+2 \epsilon)-\psi(1+\epsilon)+\psi(1-\epsilon)+\psi(1)\right)\left(q_{1}^{2 \epsilon}+q_{2}^{2 \epsilon}-q^{2 \epsilon}\right) \\
\left.+\frac{1}{2}\left(\frac{1}{\epsilon}+2 \psi(1+2 \epsilon)-2 \psi(1+\epsilon)+2 \psi(1-\epsilon)-2 \psi(1)\right) q^{2 \epsilon}-\epsilon K_{1}\right]-\beta N \frac{2 \epsilon}{(1+2 \epsilon)(3+2 \epsilon)}\left(\chi\left(q_{1}\right)+\chi\left(q_{2}\right)-\chi(q)\right),
\end{gathered}
$$

where, as an expansion in powers of $\epsilon$,

$$
K_{1}=\frac{1}{2} q^{2 \epsilon}\left[\frac{1}{\epsilon^{2}}\left(2-\left(\frac{q_{1}^{2}}{q^{2}}\right)^{\epsilon}-\left(\frac{q_{2}^{2}}{q^{2}}\right)^{\epsilon}\right)+4 \psi^{\prime \prime}(1) \epsilon+\ln \left(\frac{q_{1}^{2}}{q^{2}}\right) \ln \left(\frac{q_{2}^{2}}{q^{2}}\right)\right] .
$$

Summing the three contribution we find the final NLO gluon impact factor as

$$
\begin{gathered}
\Phi_{g}^{(1)}\left(q, q_{1}\right)=\beta \Phi_{g}^{(0)} N\left[\left(q_{1}^{2 \epsilon}+q_{2}^{2 \epsilon}\right)\left(\frac{1}{2 \epsilon}+\frac{11+7 \epsilon}{2(1+2 \epsilon)(3+2 \epsilon)}-\psi(1+\epsilon)+\psi(1+2 \epsilon)\right)+\epsilon K_{1}\left(q, q_{1}\right)+\right. \\
\left.q^{2 \epsilon}\left(\log \frac{s_{0}}{q^{2}}+\frac{1}{\epsilon}-\frac{11+8 \epsilon}{(1+2 \epsilon)(3+2 \epsilon)}-2 \psi(1+2 \epsilon)+2 \psi(1)\right)\right]- \\
-\beta \Phi_{g}^{(0)} n_{f}\left[\left(q_{1}^{2 \epsilon}+q_{2}^{2 \epsilon}\right) \frac{1+\epsilon}{(1+2 \epsilon)(3+2 \epsilon)}-q^{2 \epsilon} \frac{2(1+\epsilon)^{2}+\epsilon}{(1+\epsilon)(1+2 \epsilon)(3+2 \epsilon)}\right] \\
+\beta \chi(q)\left[-n_{f} \frac{2 \epsilon}{(1+\epsilon)(1+2 \epsilon)(3+2 \epsilon)}+N \frac{2 \epsilon}{(1+2 \epsilon)(3+2 \epsilon)}\right] .
\end{gathered}
$$




\section{The quark impact factor in the gluon colour channel}

The quark NLO impact factor is a sum of two contributions coming from the quark intermediate state and the quark-gluon intermediate state. The contribution from the quark intermediate state can be written as

$$
\begin{aligned}
\Phi_{q 1}^{(1)} /\left(\beta \Phi_{q}^{(0)}\right) & =N q_{1}^{2 \epsilon} \ln \left(\frac{s_{0}}{q_{1}^{2}}\right)+N q_{2}^{2 \epsilon} \ln \left(\frac{s_{0}}{q_{2}^{2}}\right)+\left(q_{1}^{2 \epsilon}+q_{2}^{2 \epsilon}\right)\left\{-n_{f} \frac{(1+\epsilon)}{(1+2 \epsilon)(3+2 \epsilon)}-\frac{1}{N}\left[\frac{1}{\epsilon(1+2 \epsilon)}+\frac{1}{2}\right]\right. \\
+ & \left.N\left[\psi(1-\epsilon)-2 \psi(\epsilon)+\psi(1)+\frac{1}{4(1+2 \epsilon)(3+2 \epsilon)}-\frac{1}{\epsilon(1+2 \epsilon)}-\frac{7}{4(1+2 \epsilon)}\right]\right\} . \quad(26)
\end{aligned}
$$

Here $\Phi_{q}^{(0)}$ is the LO quark impact factor in the gluon colour channel

$$
\Phi_{q}^{(0)}=-i g^{2} \frac{\sqrt{N}}{2} t \delta_{\lambda^{\prime} \lambda}
$$

with $t$ the quark colour and $\lambda$ 's its helicities. The contribution from the quark-gluon intermediate state can be written as

$$
\begin{aligned}
& \Phi_{q 2}^{(1)}\left(q, q_{1}\right) /\left(\beta \Phi_{q}^{(0)}\right)=-\frac{N}{2}\left[-4 q^{2 \epsilon}\left(\frac{1}{2} \ln \left(\frac{s_{0}}{q^{2}}\right)+\psi(1)-\psi(1+2 \epsilon)-\frac{3}{4(1+2 \epsilon)}\right)\right. \\
& +\left(q_{1}^{2 \epsilon}+q_{2}^{2 \epsilon}\right)\left(-\frac{1}{\epsilon}-\frac{3}{1+2 \epsilon}+2 \psi(1-\epsilon)-2 \psi(1+2 \epsilon)+2 \psi(1)-2 \psi(\epsilon)\right) \\
& \left.+2 q_{1}^{2 \epsilon} \ln \left(\frac{s_{0}}{q_{1}^{2}}\right)+2 q_{2}^{2 \epsilon} \ln \left(\frac{s_{0}}{q_{2}^{2}}\right)-2 \epsilon K_{1}\right]+\frac{1}{N}\left[\frac{1}{\epsilon(1+2 \epsilon)}+\frac{1}{2}\right]\left(q_{1}^{2 \epsilon}+q_{2}^{2 \epsilon}-q^{2 \epsilon}\right) .
\end{aligned}
$$

Summing these two terms we find the quark NLO impact factor in the gluon channel as

$$
\begin{gathered}
\Phi_{q}^{(1)}\left(q, q_{1}\right)=\beta \Phi_{q}^{(0)}\left\{( q _ { 1 } ^ { 2 \epsilon } + q _ { 2 } ^ { 2 \epsilon } ) \left[N\left(\frac{1}{2 \epsilon}+\frac{11+7 \epsilon}{2(1+2 \epsilon)(3+2 \epsilon)}-\psi(1+\epsilon)+\psi(1+2 \epsilon)\right)\right.\right. \\
\left.-n_{f} \frac{1+\epsilon}{(1+2 \epsilon)(3+2 \epsilon)}\right]+N \epsilon K_{1}\left(q, q_{1}\right)+ \\
q^{2 \epsilon}\left[N\left(\log \frac{s_{0}}{q^{2}}-\frac{3}{2(1+2 \epsilon)}+2 \psi(1)-2 \psi(1+2 \epsilon)\right)-\frac{1}{N}\left(\frac{1}{\epsilon(1+2 \epsilon)}+\frac{1}{2}\right)\right\} .
\end{gathered}
$$

\section{The gluonic wave function and the NLO bootstrap}

Inspecting Eqs. (25) and (29) we conclude that requirement (14) following from the third bootstrap condition is fulfilled. Indeed the parts of the NLO correction to the ratio $\Phi / \Phi^{(0)}$ which depend on the gluonic momenta $q_{1}$ and $q_{2}$ are identical in the gluon and quark impact factors. If a stronger condition (8) is obeyed, this allows to find the gluon wave function in the NLO:

$$
\Psi_{0}\left(q, q_{1}\right)=1+a\left(q_{1}^{2 \epsilon}+q_{2}^{2 \epsilon}\right)+\beta N \epsilon K_{1}\left(q, q_{1}\right)
$$

where

$$
a=\beta\left[N\left(\frac{1}{2 \epsilon}+\frac{11+7 \epsilon}{2(1+2 \epsilon)(3+2 \epsilon)}-\psi(1+\epsilon)+\psi(1+2 \epsilon)\right)-n_{f} \frac{1+\epsilon}{(1+2 \epsilon)(3+2 \epsilon)}\right]
$$

and $\beta$ and $K_{1}$ are given by (21) and (24) respectively. Of course, arbitrary NLO terms depending only on $q$ can be added to (30), but this only influences the normalization of the wave function. It is remarkable that the gluon wave function remains infrared finite also in the NLO. Indeed in the limit $\epsilon \rightarrow 0$ one gets from (30) and (31) (dropping all terms independent of $q_{1}$ and $q_{2}$ )

$$
\Psi_{0}=1-\frac{g^{2}}{32 \pi^{2}}\left[\left(\frac{11}{3} N-\frac{2}{3} n_{f}\right)\left(\ln q_{1}^{2}+\ln q_{2}^{2}\right)+N\left(\ln q_{1}^{2} \ln q_{2}^{2}-\ln q^{2} \ln q_{1}^{2}-\ln q^{2} \ln q_{2}^{2}\right)\right]
$$


It is instructive to compare (30) with the form (16) which follows from the anzatz (10). The part of $\xi$ which comes from the quarks (that is, proportional to $n_{f}$ ) was found in [3] to be

$$
\xi(q)=2 n_{f} \beta q^{2 \epsilon} \frac{1+\epsilon}{(1+2 \epsilon)(3+2 \epsilon)} .
$$

From (30) and (31) we observe that the quark contribution to the wave function is exactly given by (16). So the ansatz (10) works in the quark sector. However, if (8) is true, it does not work in the gluonic sector. The part of the gluon function coming from gluon intermediate states contains function $K_{1}\left(q, q_{1}\right)$, which cannot be represented in a simple form (16), since it mixes dependence on $q, q_{1}$ and $q_{2}$. In the limit $\epsilon \rightarrow 0$ ansatz (10) gives only the first term in the square brackets in (32) (linear in logarithms) the second term, quadratic in the logarithms, missing.

Note that the term linear in logarithms, in fact, provides for the running in the coupling. Multiplying (32) by $g^{2}$ one presents the gluon wave function up to the NLO in the form

$$
\Psi_{0}\left(q, q_{1}\right)=g^{2}\left(q_{1} q_{2}\right)\left(1-\frac{g^{2}\left(q_{1} q_{2}\right) N}{32 \pi^{2}}\left(\ln q_{1}^{2} \ln q_{2}^{2}-\ln q^{2} \ln q_{1}^{2}-\ln q^{2} \ln q_{2}^{2}\right)\right) .
$$

where

$$
g\left(q_{1} q_{2}\right)=g^{2}\left[1-\frac{g^{2}}{16 \pi^{2}}\left(\frac{11}{3} N-\frac{2}{3} n_{f}\right) \ln q_{1} q_{2}\right]
$$

is a running coupling constant at scale $q_{1} q_{2}$. The running of the coupling and scale are correctly reproduced by ansatz (10). However corrections of the 2nd order in the running coupling are missing.

Knowledge of the NLO wave function of the gluon allows to write the main bootstrap condition (the "first" one) in the NLO as an identity to be satisfied by the NLO potential at all $q$ and $q_{1}$, similar to its form in the LO:

$$
\left(H^{(0)}+\omega^{(0)}\right) \Psi_{0}^{(1)}+\left(H^{(1)}+\omega^{(1)}\right) \Psi_{0}^{(0)}=0 .
$$

Here

$$
H=-\omega\left(q_{1}\right)-\omega\left(q_{2}\right)-V_{12}
$$

is the 2-gluon Hamiltonian in the gluon colour channel. Upper indeces denote the LO (0) and NLO (1). In a more explicit form (36) reads:

$$
\begin{gathered}
\int \frac{d^{2} q_{1}^{\prime}}{{q_{1}^{\prime 2}}^{\prime 2}{ }^{2}} V_{12}^{(1)}\left(q_{1}, q_{2} \mid q_{1}^{\prime}, q_{2}^{\prime}\right)=\omega^{(1)}(q)-\omega^{(1)}\left(q_{1}\right)-\omega^{(1)}\left(q_{2}\right)+ \\
\left(\omega^{(0)}(q)-\omega^{(0)}\left(q_{1}\right)-\omega^{(0)}\left(q_{2}\right)\right)\left(a\left(q_{1}^{2 \epsilon}+q_{2}^{2 \epsilon}\right)+\beta N \epsilon K_{1}\left(q, q_{1}\right)\right)- \\
\frac{g^{2} N}{16 \pi^{3}} \int \frac{d^{2} q_{1}^{\prime}}{{q_{1}^{\prime 2} q_{2}^{\prime 2}}^{2}}\left(\frac{q_{1}^{2}{q_{2}^{\prime}}^{2}+q_{2}^{2}{q_{1}^{\prime}}^{2}}{\left(q_{1}-q_{1}^{\prime}\right)^{2}}-q^{2}\right)\left(a\left(q_{1}^{\prime 2 \epsilon}+{q_{2}^{\prime}}^{2 \epsilon}\right)+\beta N \epsilon K_{1}\left(q, q_{1}^{\prime}\right)\right) .
\end{gathered}
$$

Since the part of the NLO potential coming from the quark agrees with the anzatz (10), as found in [3], we expect that this part satisfies (38). For the still unknown gluonic part Eq. (38) presents a highly non-trivial condition to be satisfied at all $q$ and $q_{1}$.

Note that considering (36) as an equation for the NLO part $\Psi_{0}^{(1)}$ one evidently finds a requirement that the inhomogeneous (2nd) term should be orthogonal to the solution of the homogeneous equation, which is $\Psi_{0}^{(0)}=1$. So one gets a condition

$$
\left\langle\Psi_{0}^{(0)}\left|H^{(1)}+\omega^{(1)}(q)\right| \Psi_{0}^{(0)}\right\rangle,
$$

which was first obtained in [7]. Relation (39) also gives a condition to be satisfied by the NLO potential. However it is much less stringent as compared to (38), since it is only an identity in $q$, the dependence on $q_{1}$ having been integrated out.

\section{Acknowledgments.}

The authors express their deep gratitude to Prof. G.Venturi for his constant interest in this work. They thank the INFN and Physics Department of Bologna University for their hospitality and financial support. G.P.V. gratefully acknowledges the financial support from the Alexander von Humboldt Stiftung. 


\section{References.}

1.V.S.Fadin, R.Fiore, M.I.Kotsky and A.Papa, preprint BUDKERINP/99-61, UNICAL-TH 99/3 (hepph/9908264).

2.V.S.Fadin, R.Fiore, M.I.Kotsky and A.Papa, preprint BUDKERINP/99-62, UNICAL-TH 99/ hepph/9908265).

3. M.A.Braun and G.P.Vacca, Phys. Lett. 454 (1999) 319.

4. L.N.Lipatov, Yad. Fiz. 23 (1976) 642; J.Bartels, Nucl. Phys. B151 (1979) 293.

5. M.A.Braun, Phys. Lett. B345 (1995) 155; B348 (1995) 190.

6. V.S.Fadin, R.Fiore and A.Papa, Phys. Rev. D 60 (1999) 074025.

7. V.S.Fadin, R.Fiore, Phys. Lett. B 440 (1998) 359. 